\title{
Contribuciones en años de vida por sexo y edad en México
}

\begin{abstract}
Alejandro Mina Valdés*
Los logros en materia de salud de la población mexicana, sin duda, se reflejan en los incrementos que en tiempo de vida llega a obtener en su estructura por edad. En este trabajo se estiman las ganancias en la esperanza de vida o años que en promedio vive una persona a partir de cada una de las edades contempladas en la estructura de la población. esto por sexo y edad a nivel nacional y en el periodo 1950 a 1980 . Se comenta para cada edad los cambios sufridos en dichas ganancias y se resaltan las diferencias entre hombres y mujeres. Se hace una presentación exhaustiva del método de Pollard, el cual permite conocer ios alcances y limitaciones de la metodología empleada para cuantificar las ganancias en las esperanzas de vida. También se presentan gráficamente las diferencias por sexo y para cada edad, de los que se obtienen así, las distribuciones de los aumentos en los años de vida, esto último entre 1950 y 1980.
\end{abstract}

\section{Introducción}

Una de las metas más importantes de tóda política de salud es el de incrementar los años de vida que se espera viva su población, esto particularmente visto por edad y sexo de las personas objeto de acción de la política de salud establecida.

La finalidad de la presente investigación es dar un diagnóstico de las ganancias en las esperanzas de vida por edad que ha logrado la población mexicana a partir de 1950, cuando se inician los grandes logros en cuanto a la reducción de los niveles de mortalidad que imperaban en nuestro país.

El análisis se presenta a nivel nacional, por sexo y para las edades comprendidas en toda tabla abreviada de mortalidad, es decir: $0,1,5,10, \ldots, 75$ y 80 años de edad. Debe destacarse que las tablas empleadas fueron evaluadas y corregidas empleando uno de los métodos más modernos para su obtención (véase Mina, 1987). En dichas tablas también fue posible considerar y corregir el subregistro de la mortalidad, tanto infantil como adulta en México (véase Mina, 1988).

\footnotetext{
* Profesor-investigador del Centro de Estudios Demográficos y de Desarrollo Urbano de El Colegio de México.
} 
Una vez obtenidas y estimadas las tablas abreviadas de mortalidad, por ende las esperanzas de vida por sexo y por edades, se procedió a la estimación de las ganancias en las esperanzas de vida. Para ello se empleó el método de Pollard (véase Pollard, 1986). Dicho método es presentado para que el lector considere las hipótesis en que se basa y las posibles modificaciones que podrían hacérsele.

Después de mostrar la metodología empleada se presentan los resultados obtenidos y, finalmente, se da la interpretación y conclusiones a las que se llegó.

En otros trabajos (véase la bibliografía) se han señalado los logros que en materia de salud han permitido el abatimiento del impacto de las diversas causas de muerte que han dado como resultado las ganancias en la esperanza de vida que aquí se estiman.

Si bien se presenta el método de Pollard con las desagregaciones consideradas básicas para la comprensión de su alcance y limitaciones, es recomendable para posibles dudas, sobre todo de otras aplicaciones del método en el estudio de la mortalidad por causas, el profundizar en sus modalidades (véase Pollard, 1986).

Este artículo pretende ser una aportación en el estudio de la mortalidad en México y especialmente en la estimación de los niveles y tendencias de las ganancias en la esperanza de vida, esto dentro del análisis demográfico; es decir en la cuantificación del impacto de la mortalidad en México; se espera que constituya un apoyo en futuras investigaciones que se centren en la explicación de los determinantes asociados a las ganancias aquí presentadas.

\section{Presentación del método de Pollard 1}

Al considerar una disminución de magnitud $\phi$ en la fuerza de mortalidad en el intervalo de edad $x, x+h$, y suponer el mismo efecto de la mortalidad en el resto de las edades, entonces los sobrevivientes a partir de la edad $\mathrm{x}+\mathrm{h}$ de la tabla de vida se pueden estimar, en el caso discreto, partiendo del siguiente supuesto:

$$
l_{x}^{2}=l_{x}^{1}(1+\phi \mathrm{h})
$$

1 Véase Pollard, John. H. (1982), "The expectation of Life and its Relationship to Mortality". Journal of the Institute of Actuaries, vol. 109, pp. 225-240. The Alden Press Oxford. 
donde:

$l_{x}^{1}$ son los sobrevivientes a edad $x$ de la tabla de mortalidad en el momento ${ }_{i}\left({ }_{i}=1,2\right)$.

Calculando los años-persona vividos entre las edades $x \mathrm{y}_{x+5}$ :

$$
{ }_{3} L_{x}^{2}=5 / 2\left(l_{x}^{2}+l_{x+5}^{2}\right)
$$

Sustituyendo (1) en (2):

$$
\begin{aligned}
{ }_{5} L_{x}^{2} & =5 / 2\left(l_{x}^{1}+l_{x}^{1}+3\right)(1+5 \phi) \\
& ={ }_{5} L_{x}^{1}(1+5 \phi)
\end{aligned}
$$

Por lo tanto el cálculo de $T_{x}^{i}$ (años-persona acumulados a partir de la edad $x$ y hasta el final de la tabla $i_{i}={ }_{1,2}$ ) se obtiene de la siguiente manera:

$$
\begin{aligned}
T_{x}^{2} & =\sum_{j=x / 5}^{w}{ }_{5} L_{s_{j}}^{2} \\
& =\sum_{j=x / 5}^{w}{ }_{5} L_{s_{j}}^{1}(1+5 \phi) \\
& =T_{x}^{1}(1+5 \phi) \\
\rightarrow T_{x}^{2}-T_{x}^{1} & =T_{x}^{1} 5 \phi
\end{aligned}
$$

Por otro lado, la diferencia en las esperanzas de vida al nacimiento del momento 2 y 1 se estima a partir de la definición de esperanza de vida:

$$
\begin{gathered}
{ }^{o} \mathbf{e}_{o}^{2}-{ }^{o} \mathbf{e}_{o}^{1}=[1 / \mathrm{lo}]\left(\mathrm{To}^{2}-\mathrm{To}^{1}\right] \\
{ }^{o} \mathrm{e}_{o}^{2}-{ }^{o} \mathrm{e}_{o}^{1}=\int_{o}^{w}\left(l^{1}(x) / l o\right)\left[\int_{x}^{w} l_{(x)}^{1} \mathrm{dx} / l_{(x)}^{1}\right]\left[\mu_{(x)}^{1}-\mu_{(x)}^{2}\right] \mathrm{dx} \\
=\int_{o}^{w}{ }_{x}^{1} \mathrm{p}_{o}{ }^{o} \mathbf{e}_{x}^{1}\left[\mu_{(x)}^{2}-\mu_{(x)}^{2}\right] \mathrm{d} x
\end{gathered}
$$

La expresión (11) llega a dar una buena estimación de la ganancia en la esperanza de vida cuando la magnitud en la reducción de lá fuerza de mortalidad no es brusca, ya que de serlo se perdería precisión en la estimación por el efecto de relación con los distintos grupos de edades. 
Pollard ha obtenido una expresión más exacta para estimar las ganancias en la esperanza de vida (véase Pollard, 1986), su forma básica es la siguiente:

$$
{ }^{o} \mathbf{e}_{o}^{2}-{ }^{o} \mathbf{e}_{o}^{1}=\int_{o}^{w}\left[\mu_{(x)}^{1}-\mu_{(x)}^{2}\right] w_{(x)} \mathrm{d} x
$$

donde $w_{(x)}$ hace las veces de ${ }_{x} p_{o}^{1}{ }^{o} e_{x}^{2}$ de la ecuación (11), estimando Pollard a $w_{(x)}$ ponderando para cada edad, de forma aritmética, valores de ${ }_{x} p_{o}^{i}{ }^{o} e_{x}^{i}$, es decir:

$$
w(x)=0.5\left({ }_{x} \mathrm{p}_{o}^{2} \mathrm{e}_{x}^{1}+{ }_{x} \mathrm{p}_{o}^{1}{ }^{\circ} \mathrm{e}_{x}^{2}\right)
$$

La expresión que finalmente obtiene Pollard para estimar la ganancia en la esperanza de vida al nacimiento entre los momentos 2 y 1 es:

$$
{ }^{o} \mathrm{e}_{o}^{2}-{ }^{o} \mathbf{e}_{o}^{1} \cong\left({ }_{1} \mathrm{~m}_{o}^{1}-{ }_{1} \mathrm{~m}_{o}^{2}\right) w O+\left({ }_{4} \mathrm{~m}_{1}^{1}-{ }_{4} \mathrm{~m}_{1}^{2}\right) W_{1}+\sum_{x=1}^{15}\left({ }_{5} \mathrm{~m}_{5 x}^{1}-{ }_{5} \mathrm{~m}_{5 x}^{2}\right) W_{5 x}
$$

\section{Aplicación del método de Pollard}

Con el fin de ilustrar la aplicación del método de Pollard, se presenta a continuación lo hecho para la obtención de las estimaciones de las ganancias en las esperanzas de vida en México, el caso de las mujeres en el periodo 1950 a 1955,1955 a 1960.

Las tablas de mortalidad necesarias para la estimación de las ganancias en las esperanzas de vida fueron obtenidas a partir del método de Preston y Bennett (1983) aplicado al caso de México (véase Mina, 1987). De dichas tablas se tomaron las series de los sobrevivientes $\left(l_{(x)}\right)$ y las esperanzas de vida a la edad $x\left(e_{(x)}\right)$.

Para ilustrar la estimación obtenida de las ganancias de vida, se tomaron las tablas de mortalidad de las mujeres mexicanas para los periodos 1950 a 1955 y 1955 a 1960. Las series $l(x)(1)$, $\mathrm{e}(\mathrm{x})(1)$, para el primer periodo y $\mathrm{l}(x)(2), \mathrm{e}(\mathrm{x})(2)$, para el segundo, estimadas en dichas tablas, se presentan en el cuadro 1.

Con los datos del cuadro 1 se estiman, inicialmente los valores de ${ }_{n} Q_{x}(1)$ y ${ }_{n} Q_{x}(2)$, donde:

$$
\mathrm{n} Q_{x}(\mathrm{i})=-\ln \left(\mathrm{l}_{(x+n)}^{i} / /_{(x)}^{i}\right) \quad \mathrm{i}=1,2 .
$$


CUADRO 1

México: Serie de sobrevivientes $\left(I_{(x)}\right)$ y de esperanzas de vida $\left(e_{(x)}\right)$, mujeres, 1950 a 1955,1955 a 1960

\begin{tabular}{crrrr}
\hline Edad & $I(x)(1)$ & $2(x)(1)$ & $I(x)(2)$ & $e(x)(2)$ \\
\hline 0 & 100000 & 55.49 & 100000 & 60.05 \\
1 & 90893 & 60.02 & 92956 & 63.58 \\
5 & 86511 & 58.99 & 89889 & 61.70 \\
10 & 85194 & 54.86 & 88907 & 57.36 \\
15 & 84188 & 50.49 & 88151 & 52.83 \\
20 & 82767 & 46.31 & 87055 & 48.46 \\
25 & 80942 & 42.30 & 85624 & 44.23 \\
30 & 78903 & 38.33 & 83998 & 40.04 \\
35 & 76643 & 34.38 & 82164 & 35.87 \\
40 & 74147 & 30.46 & 80017 & 31.74 \\
45 & 71374 & 26.54 & 77662 & 27.65 \\
50 & 68165 & 22.67 & 74726 & 23.64 \\
55 & 64009 & 18.98 & 70821 & 19.81 \\
60 & 58706 & 15.47 & 65680 & 16.16 \\
65 & 51478 & 12.30 & 58510 & 12.84 \\
70 & 42257 & 9.43 & 48964 & 9.85 \\
75 & 30864 & 6.99 & 36693 & 7.31 \\
80 & 18743 & 4.90 & 23020 & 5.16 \\
\hline
\end{tabular}

Fuente: Mina Valdés, Alejandro (1987).

Así, por ejemplo, para $x=20$ :

$$
\begin{aligned}
{ }_{5} Q_{20}(1) & =-\ln \left(l^{1}{ }_{(25)} / I^{1}{ }_{(20)}\right) \\
& =-\ln (80942 / 82767) \\
& =.0222966
\end{aligned}
$$

Posteriormente se calculan los valores de $w_{x}$, empleando la siguiente expresión:

$$
w_{x}=0.5(l(x)(2) e(x)(1)+l(x)(1) e(x)(2)) / 100000
$$

Por ejemplo, para $x=20$ :

$$
\begin{aligned}
w_{20} & =0.5(l(20)(2) \mathrm{e}(20)(1)+l(20)(1) \mathrm{e}(20)(2)) / 100000 \\
& =0.5((87055)(46.31)+(82767)(48.46) \\
& =40.21203
\end{aligned}
$$


Otro parámetro necesario para estimar la contribución en la esperanza de vida a cada edad es la diferencia entre la fuerza de mortalidad a la edad $x$, entre los tiempos 1 y 2 ; diferencia denotada por ${ }_{n} D_{x}$, la que es igual a:

$$
{ }_{n} D_{x}={ }_{n} Q_{x}(1)-{ }_{n} Q_{x}(2)
$$

Siguiendo el ejemplo para $x=20$ :

$$
\begin{aligned}
{ }_{5} D_{20} & =.0222966-.0165745 \\
& =.0057221
\end{aligned}
$$

Finalmente se calcula la contribución en años para la esperanza de vida a cada edad, multiplicando ${ }_{n} D_{x}$ por su respectiva $w_{x}$.

En el cuadro 2 se presentan los valores obtenidos, en cuanto a las contribuciones en las esperanzas de vida para el ejemplo que nos ocupa.

\section{Presentación de los resultados}

En el anexo se presentan las tablas generadas a partir de la aplicación del método de Pollard, esto para hombres y mujeres por separado, y se presentan en ellas las ganancias en las esperanzas de

\section{CUADRO 2}

México: Contribuciones en las esperanzas de vida, estimadas a partir del método de Pollard, mujeres, periodo 1950 a 1955, 1955 a 1960

\begin{tabular}{crrrrr}
\hline Edad & $\begin{array}{r}\text { Contribución } \\
\text { en años }\end{array}$ & $\begin{array}{r}\text { Porcentaje de } \\
\text { contribución }\end{array}$ & Edad & $\begin{array}{r}\text { Contribución } \\
\text { en años }\end{array}$ & $\begin{array}{r}\text { Porcentaje de } \\
\text { contribución }\end{array}$ \\
\hline 0 & 1.2965480 & 37.176870 & 40 & .1974485 & 5.661587 \\
1 & .9007464 & 25.827760 & 45 & .1505815 & 4.317735 \\
5 & .2317383 & 6.644803 & 50 & .1526298 & 4.376467 \\
10 & .1630122 & 4.674169 & 55 & .1452470 & 4.164774 \\
15 & .2007394 & 5.755950 & 60 & .1551238 & 4.447978 \\
20 & .2300971 & 6.597745 & 65 & .1330406 & 3.814772 \\
25 & .2283371 & 6.547277 & 70 & .1127313 & 3.232429 \\
30 & .2227912 & 6.388257 & 75 & .0784558 & 2.249620 \\
35 & .1847923 & 5.298686 & & & \\
Ganancia & & & & & \\
total & & & & 3.487512 & 100.00 \\
\hline
\end{tabular}


vida estimadas para los periodos comprendidos de 1950 a 1980 , siendo el primero de 1950-1955 a 1955-1960, y el último de 19701975 a 1975-1980.

En el conjunto de gráficas del anexo se presentan las distribuciones de las ganancias en las esperanzas de vida, por sexo y grupo de edad. Con ello se pretende ilustrar la tendencia en el tiempo de las ganancias en la esperanza de vida, así como su diferencial por sexo.

\section{Interpretación de los resultados}

Al conocer ya la distribución de las ganancias en las esperanza de vida por edad y por sexo, se da a continuación para cada edad los diferenciales por sexo y en el tiempo considerado; es decir, de 1950 a 1980.

\section{Edad 0 años}

La mayor ganancia en la esperanza de vida se obtiene para los hombres en el periodo 1950-1955 a 1955-1960, con un aumento de 1.82 años, siendo para las mujeres, en el mismo intervalo de 1.30 años, no obstante que la esperanza de vida de las mujeres es mayor que la de los hombres.

Después de ese primer periodo las ganancias en la esperanza de vida son menos significativas, teniéndose, para el siguiente periodo (1955-1960 a 1960-1965) una ganancia de los hombres de 0.62 años, contra una ganancia de las mujeres de 0.43 años. Para los periodos comprendidos entre 1960-1965 y 1970-1975, se tienen prácticamente las mismas ganancias en la esperanza de vida al nacimiento para las mujeres y hombres, aumentando de 0.58 años a 0.74 años. Para el último periodo analizado (1970-1975 a 19751980) se tiene una mayor, aunque ligera, ganancia en la esperanza de vida al nacimiento, por parte de las mujeres $(0.70$ años contra 0.66 años de los hombres).

\section{Edad 1 año}

Para el periodo comprendido entre 1950-1955 y 1955-1960 se tiene la mayor ganancia en la esperanza de vida a un año; siendo ligera la diferencia por sexo; así, se tiene una e(1) de 1.11 años para los hombres contra 0.9 años de las mujeres. 
En los dos siguientes periodos considerados (1955-1960 a 1960-1965 y 1960-1965 a 1965-1970) la diferencia se reduce pasando los hombres de una e(1) de 0.35 años a 0.33 años y las mujeres de 0.28 años a 0.33 años. Para el siguiente periodo (1965-1970 a 1970-1975) se tiene una mayor ganacia en la e(1) de las mujeres sobre la de los hombres $(0.46$ años de las mujeres contra 0.41 años de los hombres); tendencia que se conserva en el último periodo considerado, 1970-1975 a 1975-1980, siendo la ganancia en la e(1) de 0.44 años para las mujeres y de 0.35 años de los hombres.

\section{Edad 5 años}

A partir de esta edad las ganancias en la esperanza de vida se reducen significativamente, teniéndose la mayor ganancia en el primer periodo, 1950-1955 a 1955-1960, 0.25 años de los hombres y 0.23 años de las mujeres.

En el siguiente periodo, $1955-1960$ a 1960-1965, las ganancias aunque pequeñas, son ligeramente superiores para los hombres, los cuales tienen una ganancia en la e(5) de 0.085 años y las mujeres de 0.075 años.

A partir del periodo 1960-1965 a 1965-1970 y hasta el último en estudio (1970-1975 a 1975-1980), se tiene una mayor ganancia en la e(5) por parte de las mujeres, pasando de 0.10 años a 0.12 años, mientras que los hombres incrementan su ganancia de 0.080 años a 0.090 años.

\section{Edad 10 años}

La ganancia en la e(10) es prácticamente la misma para los dos primeros periodos, para hombres y mujeres, teniéndose para 1950-1955 a 1955-1960 una ganacia de 0.16 años y para 1955-1960 a 1960-1965 de 0.08 años.

En el resto de los periodos, las ganancias en e(10) son mayores para las mujeres llegando a alcanzar una ganancia de 0.09 años en el periodo 1965-1970 a 1970-1975, contra 0.07 años de los hombres en el mismo periodo, manteniéndose prácticamente el mismo nivel de ganancia en 1970-1975 a 1975-1980 para las mujeres, disminuyendo ligeramente para los hombres (0.06 años). 
Edad 15 años

A partir de esta edad se inicia un repunte en la ganancia en la esperanza de vida, tanto para los hombres como para las mujeres, esto en todos y cada uno de los periodos considerados; se estima una ganancia en la e(15) de 0.19 años de los hombres y una de 0.20 años de las mujeres, para el periodo inicial (1950-1955 a 19551960), de 0.067 años y 0.066 años para el siguiente (1955-1960 a 1960-1965) conservándose esos niveles en el periodo comprendido entre 1960 y 1970, se estima para el último periodo considerado una ganancia en la e(15) masculina de 0.076 años y una femenina de 0.11 años.

\section{Edades 20 a 40 años, hombres}

En estas edades las ganancias en la e(20), e(25), e(30), e(35) y e(40), para el caso de los hombres es esencialmente la misma en cada uno de los periodos considerados, teniéndose entre 1950 y 1960 una ganancia de 0.25 años, para despues disminuir a 0.88 años hasta el periodo 1960 a 1970 , para registrarse una ganancia en las esperanzas señaladas de 0.10 años en los últimos dos periodos registrados, los que comprenden los años 1970 a 1980.

\section{Edades 20 a 30 años, mujeres}

En las esperanzas de vida e(20), e(25) y e(30) las ganancias obtenidas por la mujeres son prácticamente las mismas en cada periodo cosiderado, si bien es cierto varían entre ellos, así en 1950-1955 a 1955-1960 la ganancia en dichas esperanzas es de 0.21 años, en 1955-1960 a 1960-1965 es de 0.075 años y para el resto de los periodos oscila entre 0.10 y 0.13 años las ganancias en las esperanzas de vida consideradas.

\section{Edad 35 y 40 años, mujeres}

Las ganancias en la esperanza de vida a los 35 y 40 años son las mismas que para las esperanzas comprendidas entre las edades 20 y 30 años, exceptuando los dos primeros periodos (1950-1955 a $1955-1960$ y $1955-1960$ a 1960-1965) en donde las ganancias en la $e(35)$ son de 0.18 años y 0.09 años, respectivamente, y para la $e(40)$ de 0.20 años y 0.043 años, respectivamente. 
Cabe señalar que las ganancias en las esperanzas de vida de los 20 a los 40 años son de hecho las mismas para hombres como para las mujeres aunque ligeramente superiores para los hombres en los dos primeros periodos y superiores para las mujeres en el resto de los periodos.

\section{Edades 45 a 60 años, hombres}

Las ganancias en las esperanzas de vida a los 45 y 50 años son de 0.21 años de 1950 a 1960 , de 0.08 de 1960 a 1970 y de 0.10 años de 1970 a 1980.

Para las esperanzas de vida a los 55 y 60 años, los hombres disminuyen sus ganancias en la esperanza de vida mientras que las mujeres las conservan (véase el siguiente párrafo), teniéndose una ganancia de 0.16 años en promedio para el periodo 1950 a 1960 , de 0.065 años de 1960 a 1970 variando de forma más marcada en los dos últimos periodos, siendo la ganancia en e(55) de 0.06 y de e(60) de 0.12 años de 1965-1970 a 1970-1975, y de 0.13 años y 0.06 años en 1970-1975 a 1975-1980, respectivamente.

\section{Edades 45 a 60 años, mujeres}

En el caso de las mujeres, las ganancias en las esperanzas de vida comprendidas en las edades 45 a 60 años, los niveles son prácticamente los mismos, teniéndose en 1950-1955 a 1955-1960 de 0.15 años, de 1955-1960 a 1960-1965 de 0.05 años, de 1960-1965 a 19651970 de 0.08 años y de 1970 a 1980 de 0.10 años.

También debe resaltarse que las ganancias son similares para los hombres y mujeres en las esperanzas de vida comprendidas entre los 45 y los 60 años.

\section{Edad 65 años}

Las ganancias en la esperanza de vida a los 65 años de edad son esencialmente las mismas para los primeros dos periodos considerados, esto para hombres y mujeres, teniéndose para el resto una mayor ganancia para las mujeres. Observándose que para 19551960 a 1960-1965 una ganancia en e(65) de 0.13 años para los hombres y mujeres, siendo de 0.052 años y 0.050 años, respectivamente, para el periodo $1955-1960$ a $1960-1965$.

De 1960-1965 a 1965-1970 la ganancia en la e(65) masculina es 
de 0.054 años contra una e(65) femenina de 0.073 años En los dos últimos periodos (1965-1970 a 1970-1975 y 1970-1975 a 1975-1980) las ganancias en e(65) sufren mínimas diferencias, así, los hombres pasan de una ganancia de 0.075 años a una de 0.073 años, mientra las mujeres pasan de una ganancia de 0.099 años a una de 0.11 años.

\section{Edad 70 años}

La tendencia en las ganancias en la esperanza de vida a los 70 años es la misma que la observada en los 65 años, aunque a menores niveles; teniéndose que para $1955-1960$ a 1960-1965 una ganancia en $\mathrm{e}(70)$ de 0.10 años para los hombres y 0.11 años para las mujeres, siendo de 0.040 años, y 0.043 años respectivamente, para el periodo 1955-1960 a 1960-1965.

En 1960-1965 a 1965-1970 la ganancia en la e(70)masculina es de 0.042 años contra una e(70) femenina de 0.064 años. En los dos últimos periodos (1965-1970 a 1970-1975 y 1970-1975 a 1975-1980) las ganancias en e(70) sufren mínimas diferencias; así, los hombres pasan de una ganancia de 0.060 años a una de 0.059 años, mientras las mujeres pasan de una ganancia de 0.088 años a una de 0.10 años.

\section{Edad 75 años}

Las más pequeñas ganancias en las esperanzas de vida se obtienen en la esperanza de vida a los 75 años, siendo en el primer periodo (1950-1955 a 1955-1960) de 0.067 años para los hombres y de 0.078 años para las mujeres, pasando a 0.028 años, los hombres y 0.030 años las mujeres en el periodo (1955-1960 a 1960-1965).

La ganancia en e(75) masculina es de 0.029 años contra 0.045 años de la femenina en los años 1960-1965 a 1965-1970. Aumentando sus ganancias, tanto hombres como mujeres en los dos últimos periodos considerados a 0.043 años los hombres y de 0.064 años a 0.072 años las mujeres.

\section{Ganancias totales}

En cuanto a la estimación de la ganancia total en las esperanzas de vida (suma de las ganancias en las esperanzas de vida en cada edad) se tiene para todos los periodos considerados una mayor ganancia de las mujeres sobre los hombres, con excepción del pri- 
mer periodo. Alcanzado su mayor ganancia en el intervalo 19501955 a 1955-1960, donde la ganancia total en la esperanza de vida fue de 4.00 años para los hombres contra 3.49 años de las mujeres; para el periodo 1955-1960 a 1960-1965 es de 1.42 años para mujeres y de 1.18 años para los hombres, siendo de 1.62 años para las mujeres y 1.37 años para los hombres en 1960-1965 a 1965-1970. Aumentando ligeramente las ganancias de ambos sexos de 19651970 a 1970-1975, con una ganancia en las esperanzas de vida estimadas en 2.07 años para las mujeres y 1.80 años para los hombres; finalmente de 1970-1975 a 1975-1980 se obtiene una ganancia de 2.08 años para las mujeres y 1.65 años de los hombres.

\section{Porcentajes de contribución}

Para ilustrar y resaltar los cambios en los porcentajes de contribución en la ganancia total de la esperanza de vida en cada grupo de edad, se presentan en el cuadro 3 los porcentajes obtenidos para el primero y último periodos considerados.

Del cuadro 3 destaca el porcentaje de contribución tenido en los primeros dos grupos de edades que ocupan más del $50 \%$ de la contribución en las ganancias en la esperanza de vida, esto para los hombres como para las mujeres. Los porcentajes de las ganancias en la esperanza de vida oscilan entre 4 y $6 \%$ para el resto de los grupos de edades, exceptuando los grupos de edades por arriba de 60 a 64 años, donde el porcentaje oscila entre 2 y 5 .

En cuanto a los diferenciales por sexo, se tiene que para los dos primeros grupos de edades y para los dos periodos considerados un mayor porcentaje para los hombres, sobre todo para la edad cero años cumplidos, teniéndose para el resto de las edades aproximadamente los mismos porcentajes obtenidos; es ligeramente mayor para las mujeres en las edades avanzadas. Al observar los porcentajes entre periodos, se observa que los hombres disminuyen sus porcentajes de contribución en la ganancia de las esperanzas de vida de los ceros años cumplidos a 35 a 39 años cumplidos, para posteriormente, aumentarlo entre los grupos de edades 40 a 44 años cumplidos a 55 a 59 años cumplidos. En el siguiente grupo de edades (60 a 64 años cumplidos) se da una muy ligera disminución en el porcentaje (de 3.96 a 3.45) para mantener su tendencia al alza en el resto de las edades.

El caso de las mujeres es diferente ya que los porcentajes disminuyen para los grupos de edades comprendidos entre los ceros años cumplidos y los 30 a 34 años y aumentan para el resto de las 
CUADRO 3

México: Porcentaje de contribución de la ganancia total en las esperanzas de vida, por grupos de edades y sexo, 1950-1955, a 1955-1960, y 1970-1975, a 1975-1980

\begin{tabular}{cccccr}
\hline & \multicolumn{2}{c}{$1950-1955$} & & \multicolumn{2}{c}{$1970-1975$} \\
Grupos de & a 1955-1960 & & \multicolumn{2}{c}{ a 1975-1980 } \\
\cline { 2 - 3 } \cline { 5 - 6 } & Hombres & Mujeres & & Hombres & Mujeres \\
\hline 0 & 45.42 & 37.18 & 39.79 & 33.85 \\
$1-4$ & 27.69 & 25.83 & & 21.74 & 21.17 \\
$5-9$ & 6.19 & 6.64 & & 5.47 & 5.82 \\
$10-14$ & 3.91 & 4.67 & & 3.66 & 4.18 \\
$15-19$ & 4.78 & 5.76 & & 4.58 & 5.16 \\
$20-24$ & 6.16 & 6.60 & & 5.91 & 5.93 \\
$25-29$ & 6.26 & 6.55 & & 5.99 & 6.06 \\
$30-34$ & 6.36 & 6.39 & & 6.11 & 6.12 \\
$35-39$ & 6.34 & 5.30 & & 6.28 & 5.90 \\
$40-44$ & 6.17 & 5.66 & & 6.44 & 5.50 \\
$45-49$ & 5.45 & 4.32 & & 6.07 & 4.91 \\
$50-54$ & 5.10 & 4.38 & & 6.04 & 5.20 \\
$55-59$ & 4.28 & 4.16 & & 7.68 & 5.17 \\
$60-64$ & 3.96 & 4.44 & 3.45 & 5.68 \\
$65-69$ & 3.22 & 3.81 & 4.44 & 5.16 \\
$70-74$ & 2.45 & 3.23 & 3.55 & 4.61 \\
$75-79$ & 1.68 & 2.25 & 2.57 & 3.44 \\
& & & & \\
\hline
\end{tabular}

Fuente: Cuadro A1 (anexo).

edades, exceptùando el grupo de edades 40 a 44 años cumplidos donde disminuye muy ligeramente (de 5.66 a 5.50 ).

\section{Conclusiones}

Las máximas ganancias en las esperanzas de vida se centran en el primer año de vida y en el grupo de edades uno a cuatro años cumplidos, siendo de más del $\mathbf{5 0} \%$ del total de las ganancias. Aunque si bien es cierto dicho porcentaje ha disminuido en el tiempo.

En la década de los cincuenta las ganancias fueron mayores en los grupos de edades intermedias con respecto a los grupos de edades avanzadas, hecho que en la década de los setenta se invirtió.

Las ganancias cada vez son menos significativas en el tiempo, exceptuando las que se obtienen en las primeras edades de la vida. 
Las mujeres mexicanas, aunque tienen una esperanza de vida mayor por edades, con respecto a los hombres, llegan a tener estas ganancias mayores en las esperanzas; lo anterior debido a que las mujeres ya han alcanzado niveles en donde obtener ganancias en las esperanzas de vida es cada vez más difícil; es decir, las ganancias en las esperanzas de vida de las mujeres tienden más a la estabilización.

Con respecto a las edades cero y uno a cuatro se tienen oportunidades en México de reducir el impacto de la mortalidad infantil y en los primeros años de vida y así poder alcanzar los niveles de estabilización que se tienen en países desarrollados, en cuanto el aporte que den a las esperanzas de vida, los que cada vez serán menores conforme se reduzcan los niveles de mortalidad infantil y en los primeros años de vida en México.

\section{Bibliografía}

Díaz A., Erwin (1987), Causas de muerte en Guatemala: 1960-1979, Centro Latinoamericano de Demografía, Serie oI. núm. 1001, San José, Costa Rica. 76 p.

Mina V., Alejandro (1987), "Elaboración de tablas de mortalidad para México, empleando el método de Preston y Bennett", Documentos de trabajo, Centro de Estudios Demográficos y de Desarrollo Urbano, El Colegio de México, $53 \mathrm{pp}$.

(1988), "Diagnóstico del registro de la mortalidad en México", Revista de Estadística, Instituto Nacional de Estadística Geografía e Informática, Secretaría de Programación y Presupuesto, pp. 33-40.

Preston S. y N.G. Bennett (1983), "A census bases method for estimating adult mortality", Population Studies, 37, pp. 91-104.

Pollard, John. H. (1986), "Cause of Death and Expectation of Life; Some International Comparisons", International Union for the Scientific Study of Population and Institute of Statistic. University of Siena. Siena, Italia, 7 al 12 de Julio.

(1982), "The expectation of Life and its Relationship to Mortality", Journal of the Institute of Actuaries. Vol. 109, pp. 225-240. The Alden Press Oxford. 


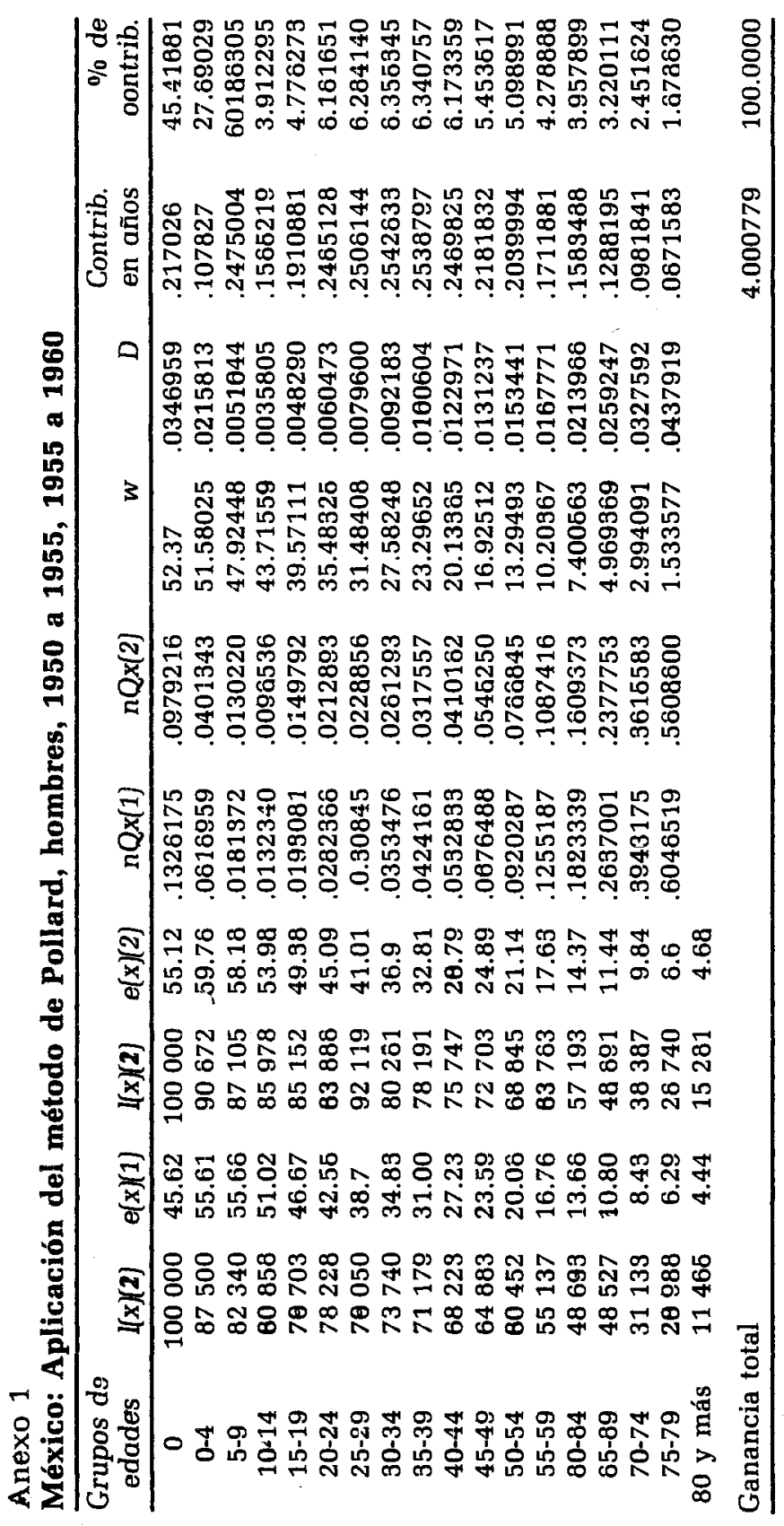




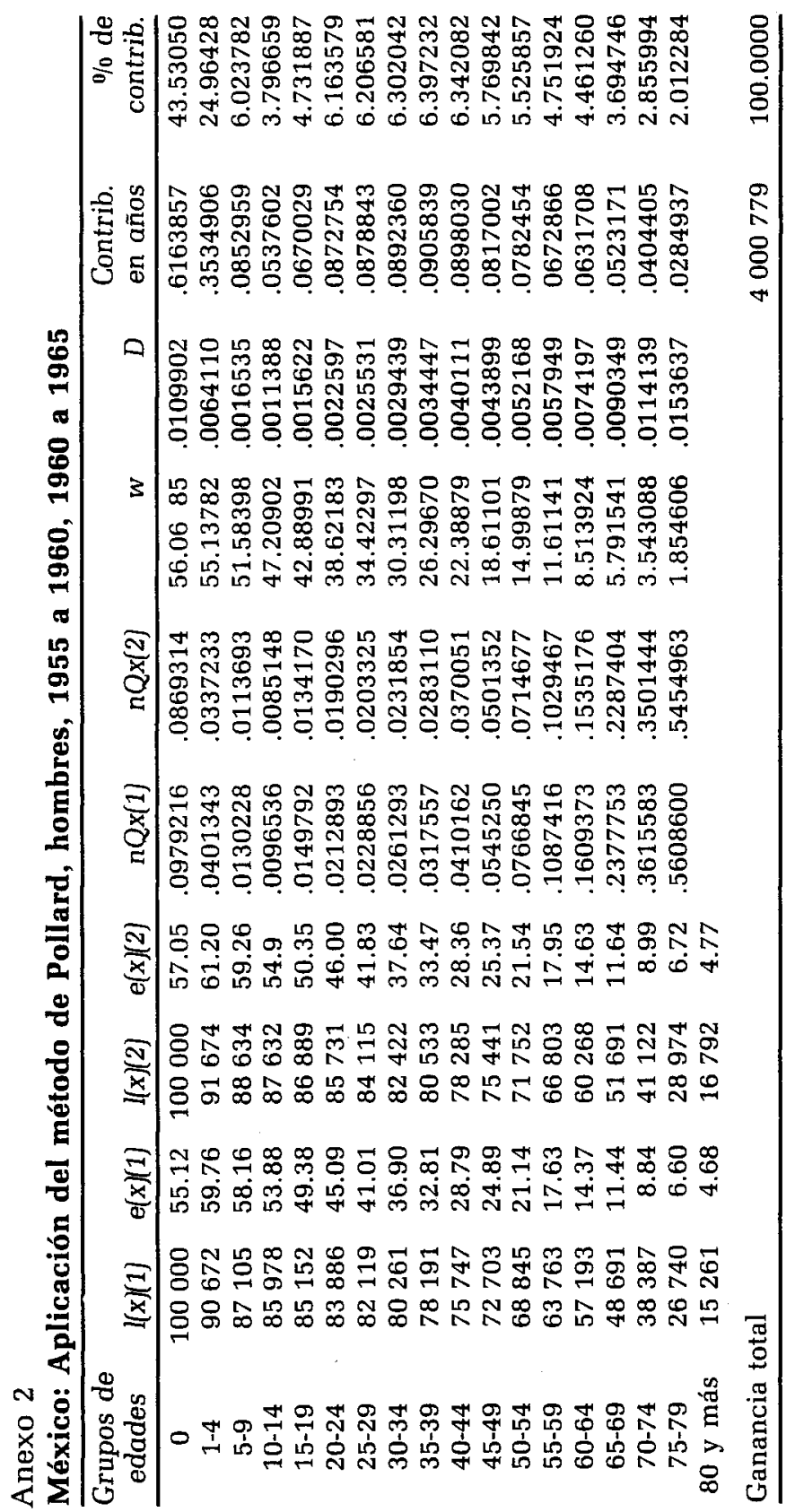




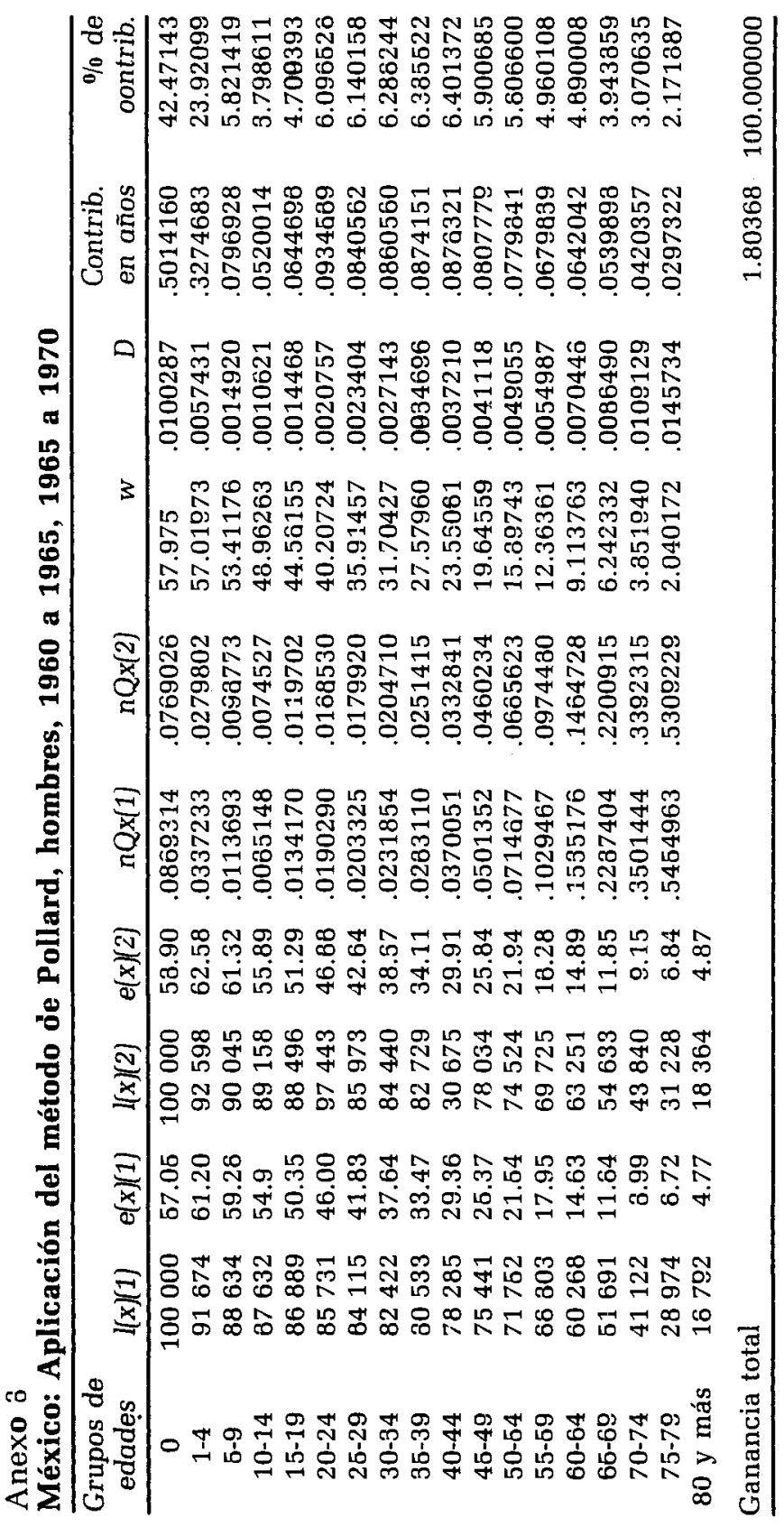




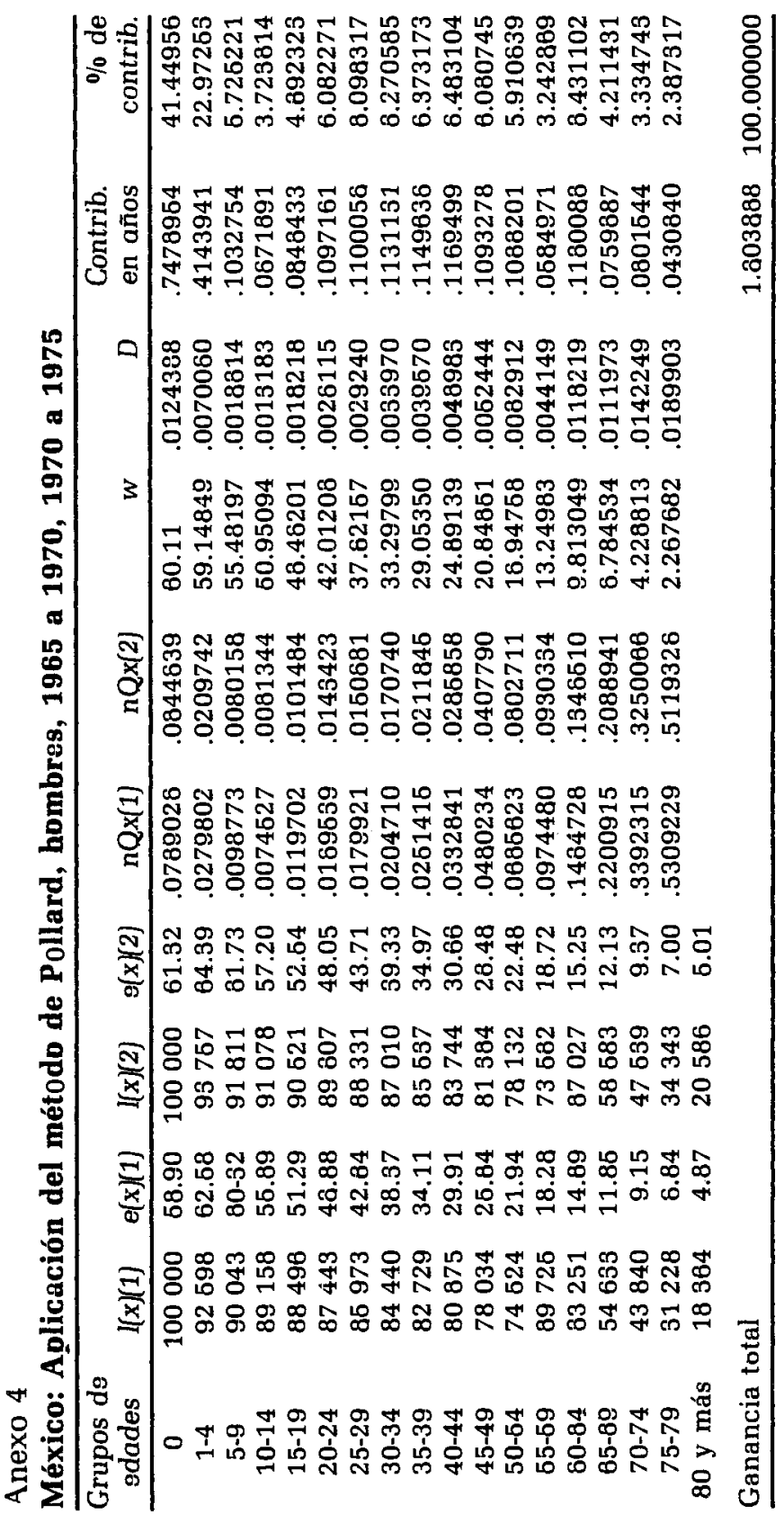




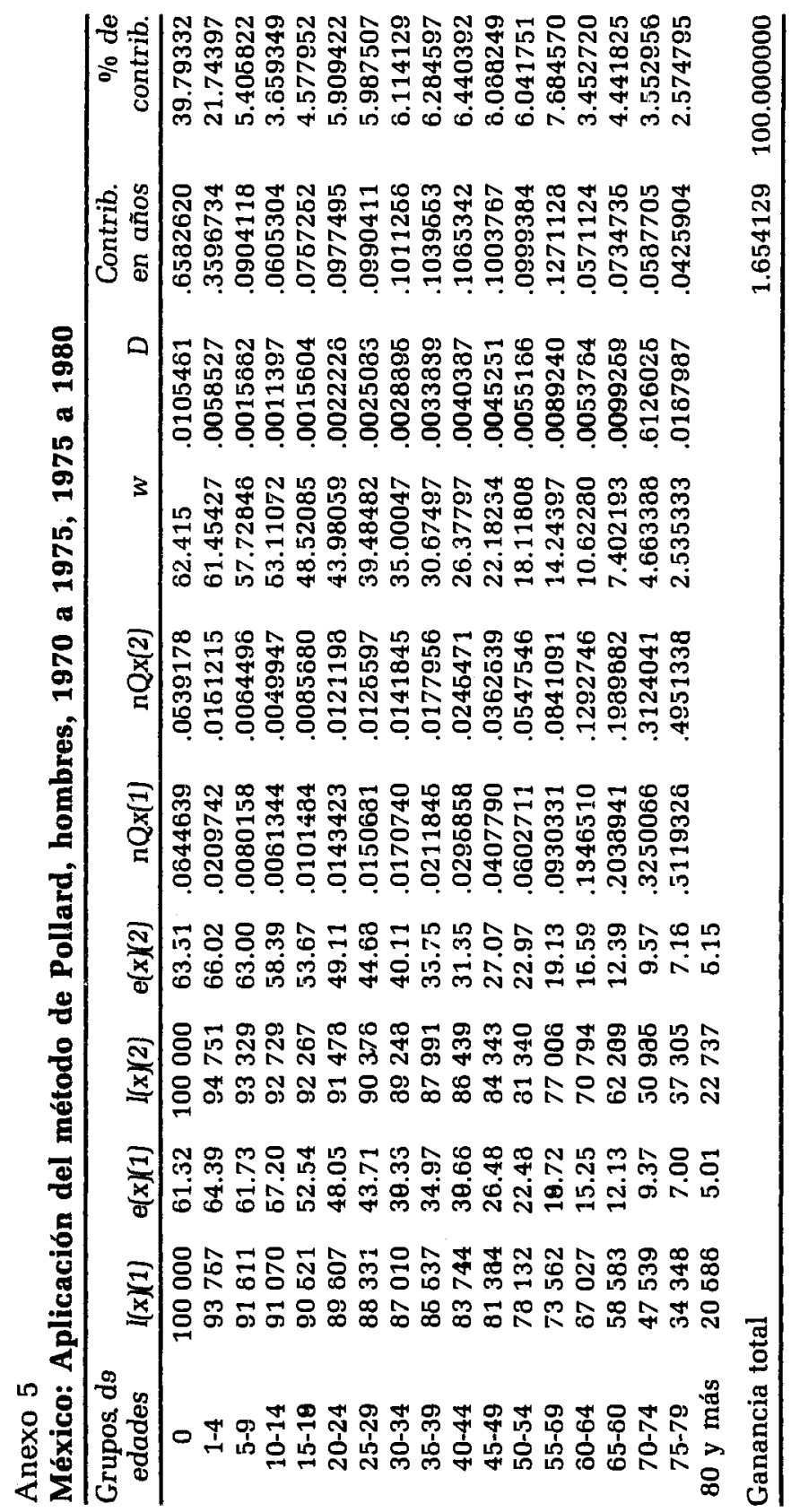




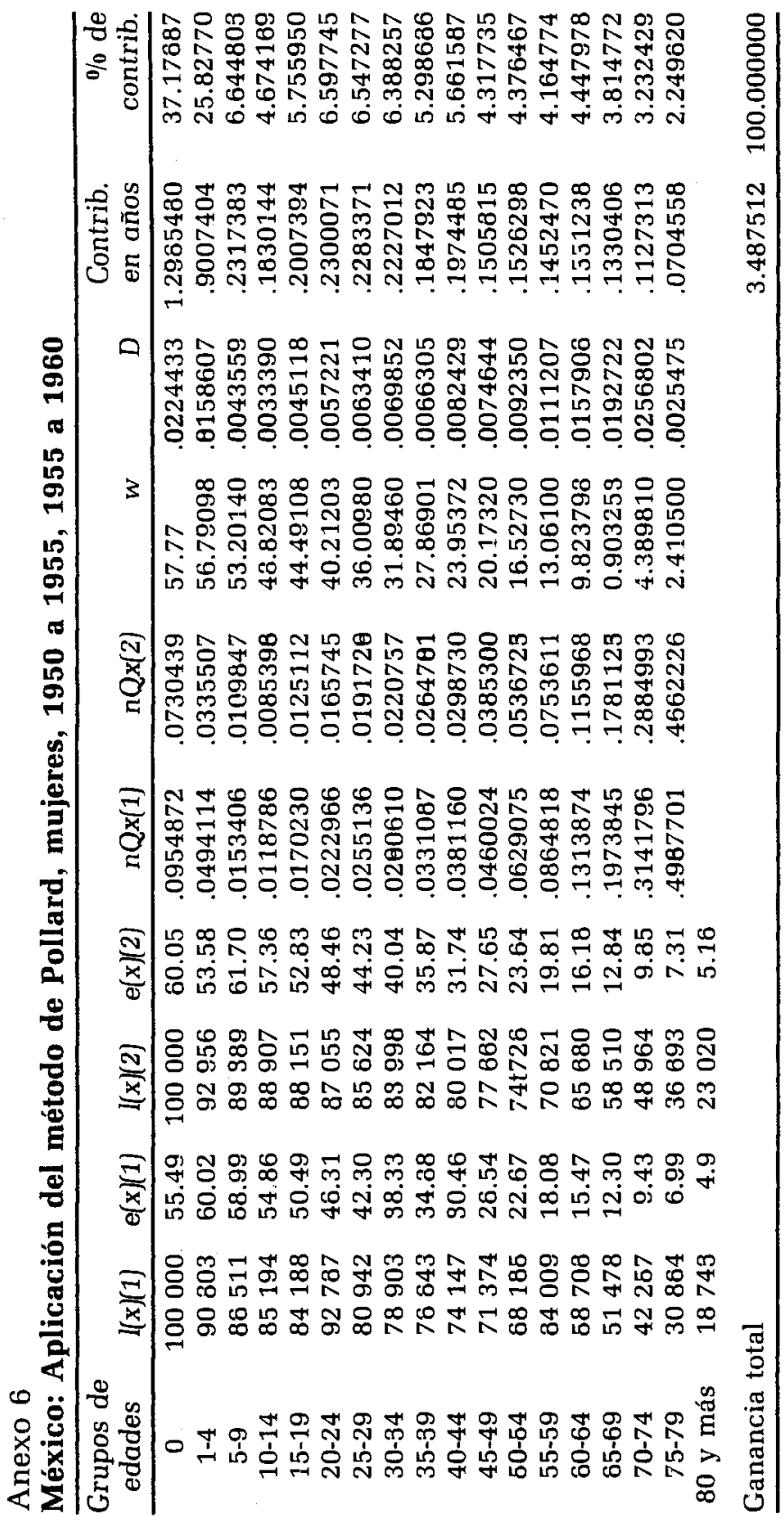




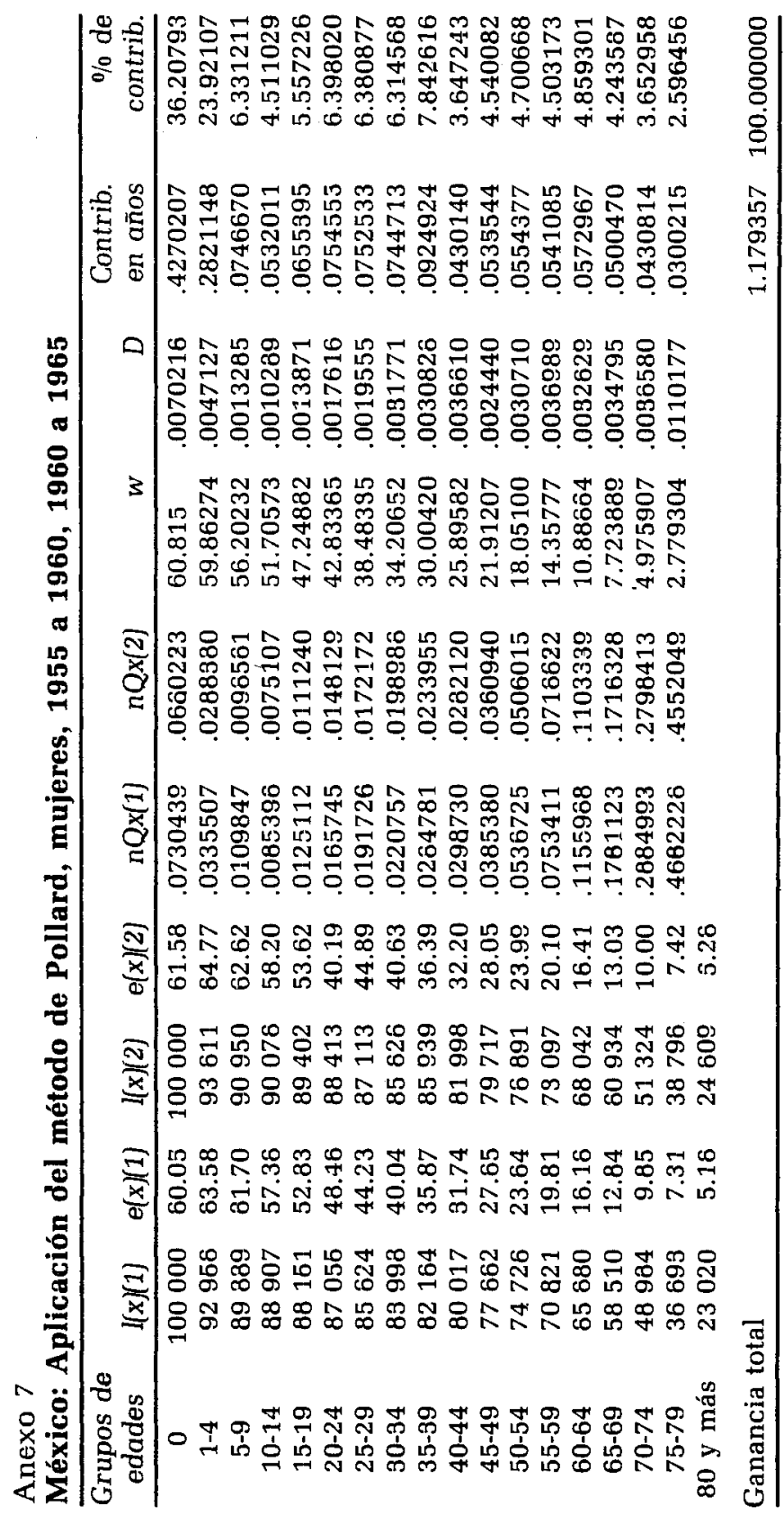




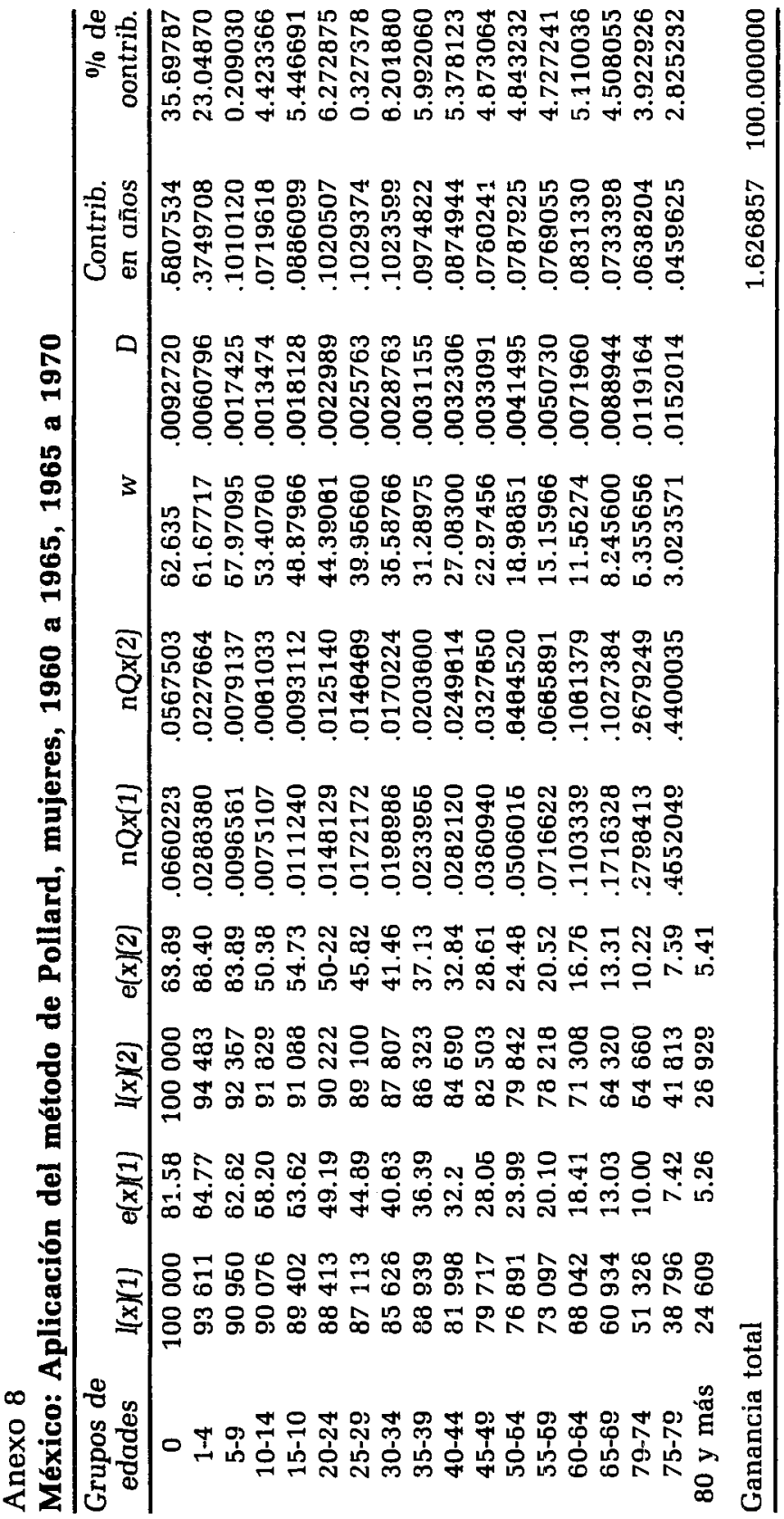




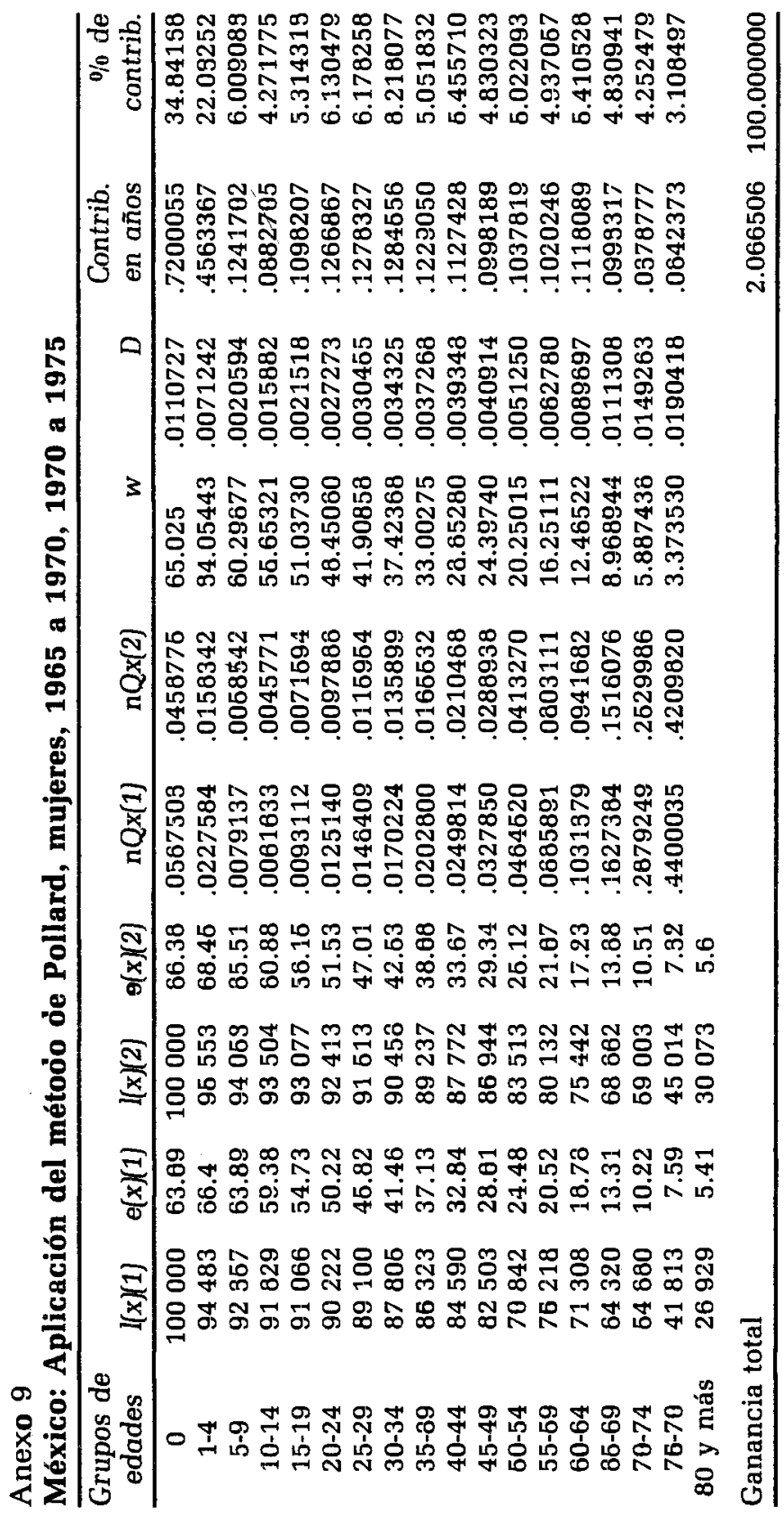




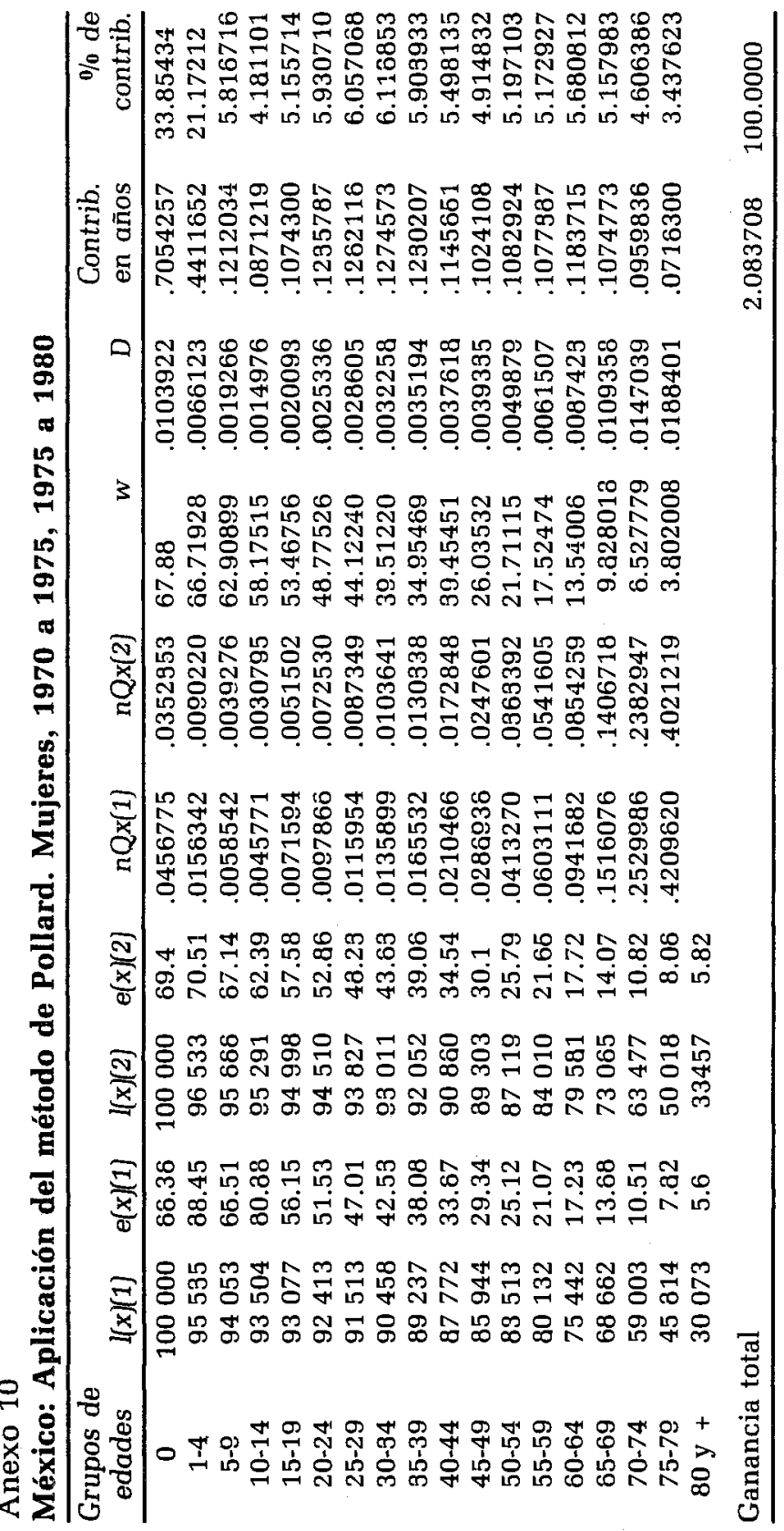


GRÁFICA 1

México: Ganancias en e(0), por sexo, 1950 a 1980

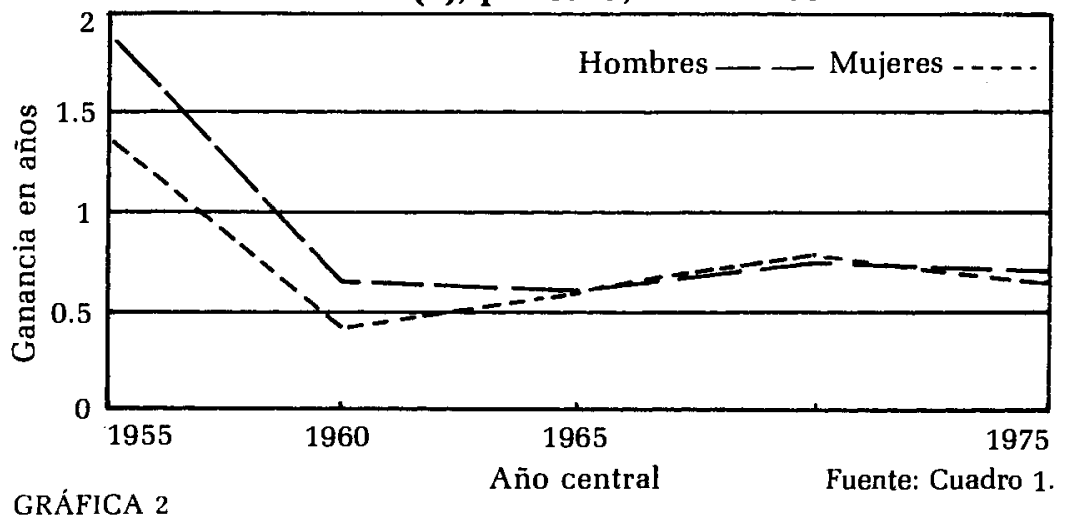

México: Ganancias en e(1), por sexo, 1950 a 1980

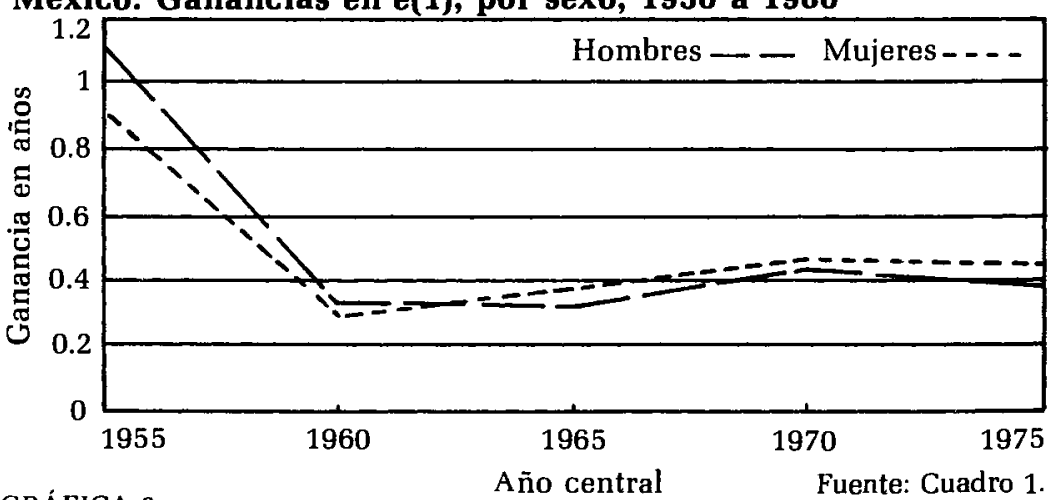

GRÁFICA 3

México: Ganancias en e(5), por sexo, 1950 a 1980

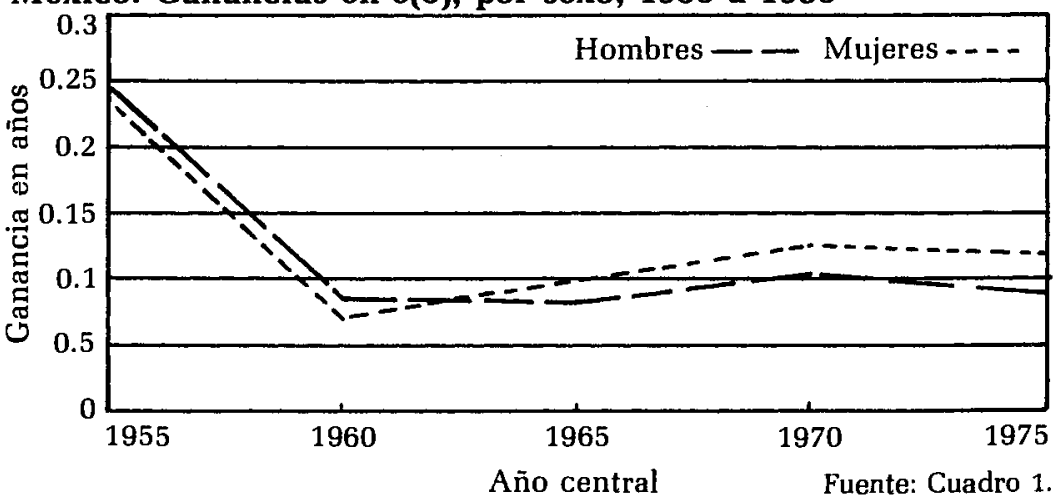




\section{GRAFICA 4}

México: Ganancias en e(10), por sexo, 1950 a 1980

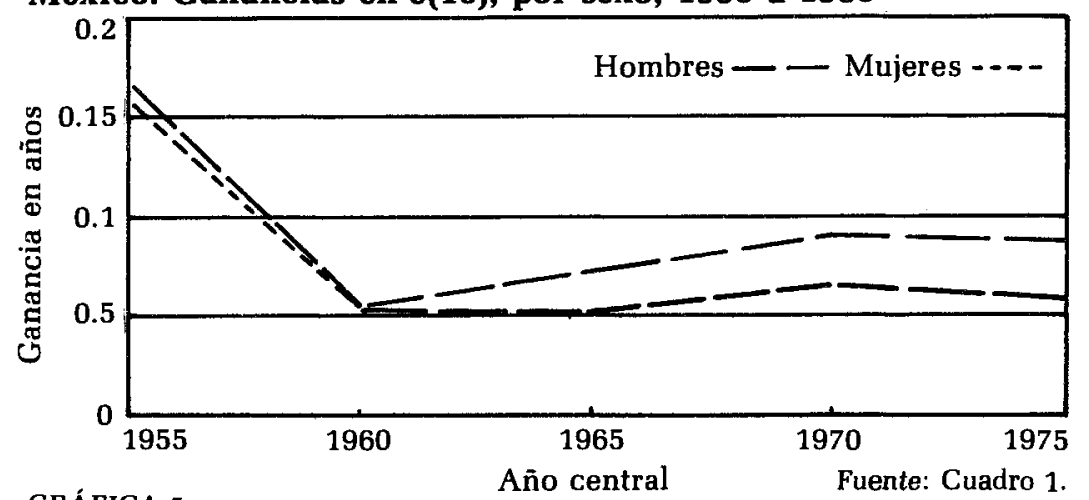

GRÁFICA 5

México: Ganancias en e(15), por sexo, 1950 a 1980

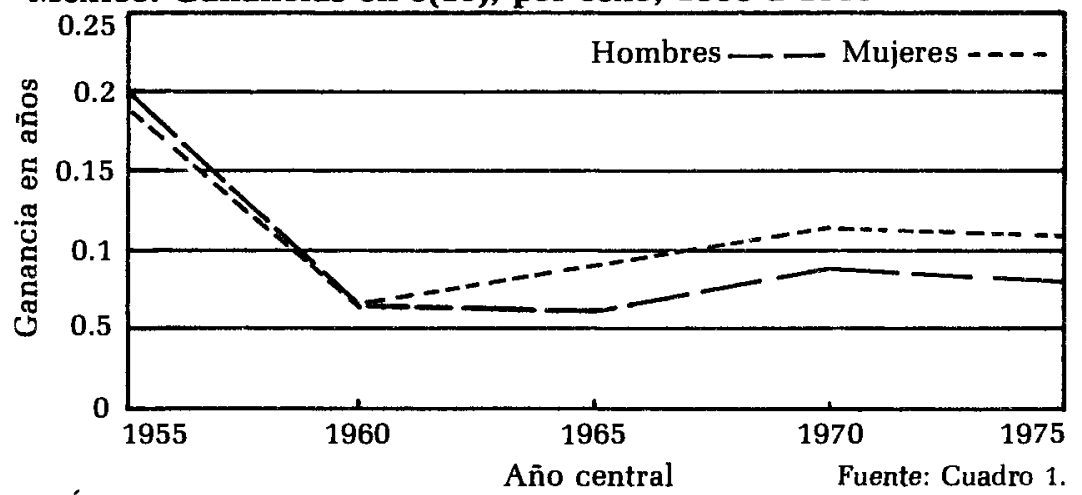

GRÁFICA 6

México: Ganancias en e(20), por sexo, 1950 a 1980

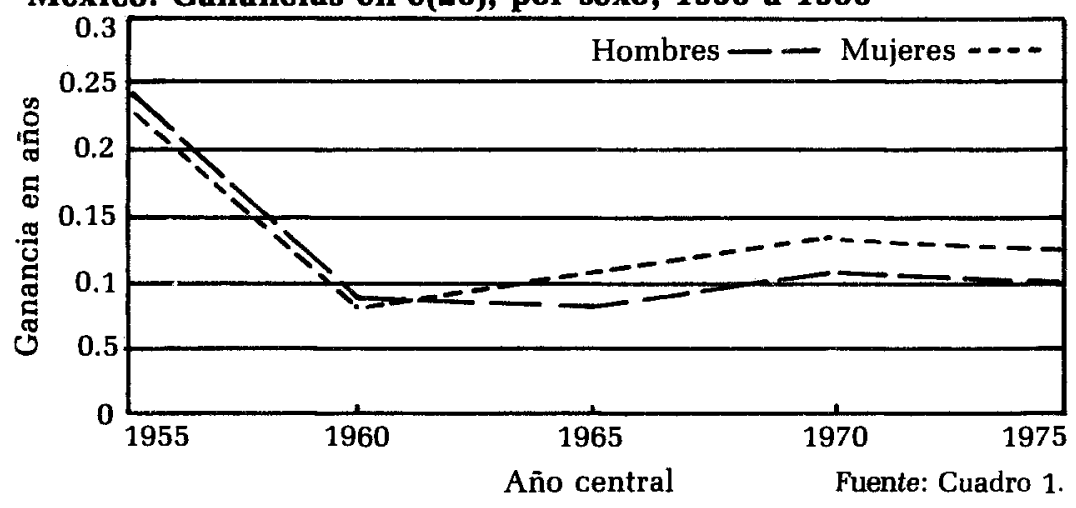


GRÁFICA 7

México: Ganancias en e(25), por sexo, 1950 a 1980

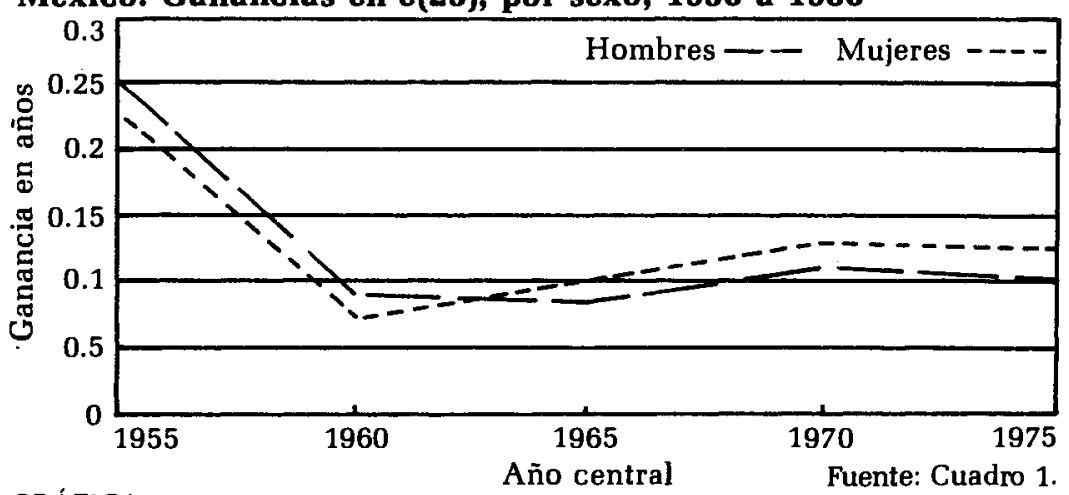

GRÁFICA 8

México: Ganancias en e(30), por sexo, 1950 a 1980

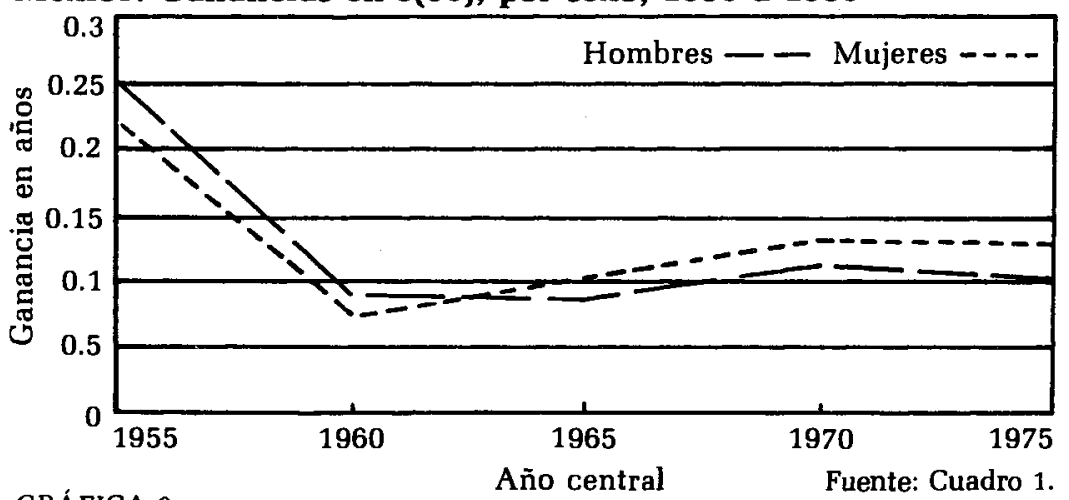

GRÁFICA 9

México: Ganancias en e(35), por sexo, 1950 a 1980

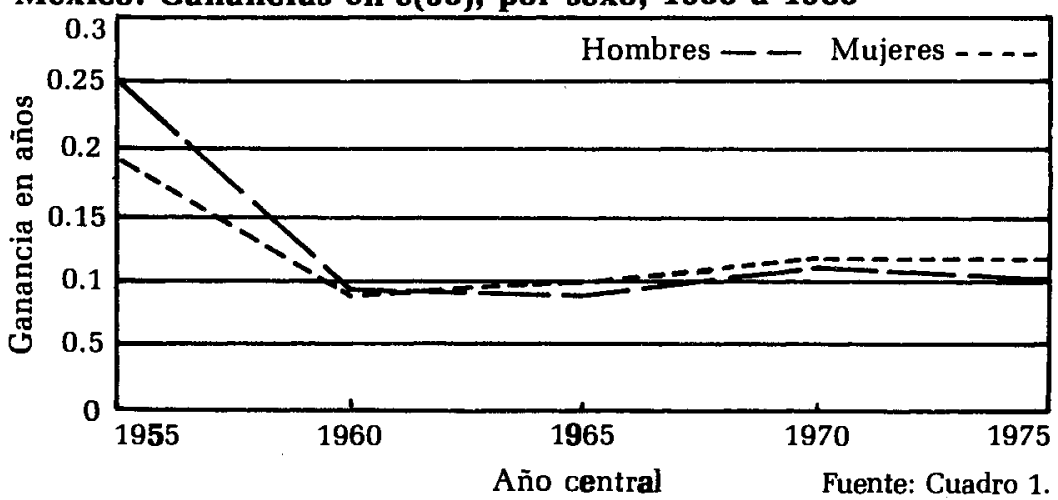


GRÁFICA 10

México: Ganancias en e(40), por sexo, 1950 a 1980

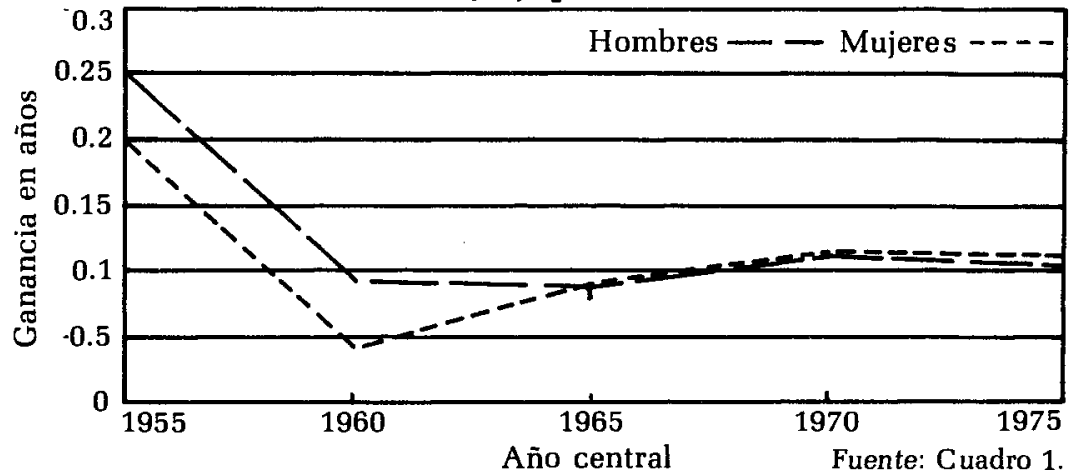

GRÁFICA 11

México: Ganancias en e(45), por sexo, 1950 a 1980

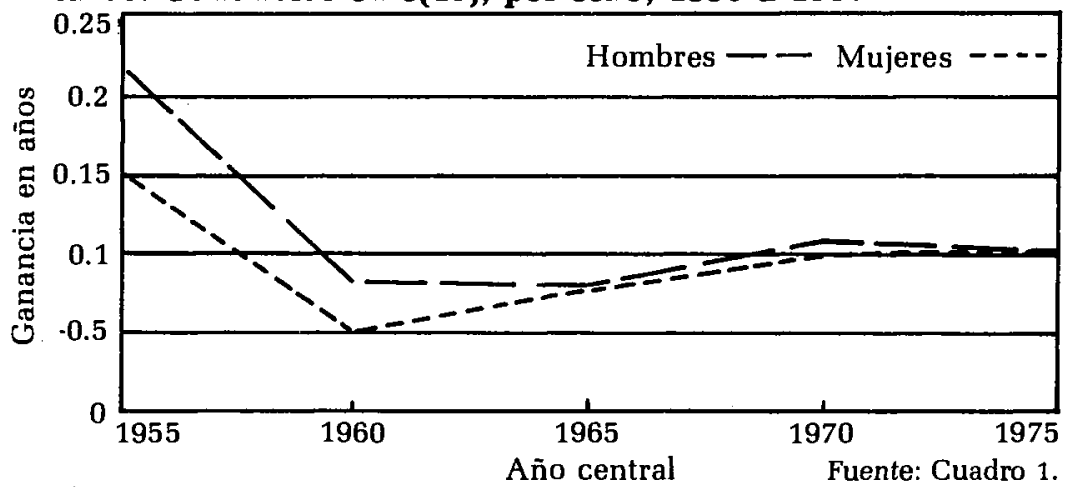

GRÁFICA 12

México: Ganancias en e(50), por sexo, 1950 a 1980

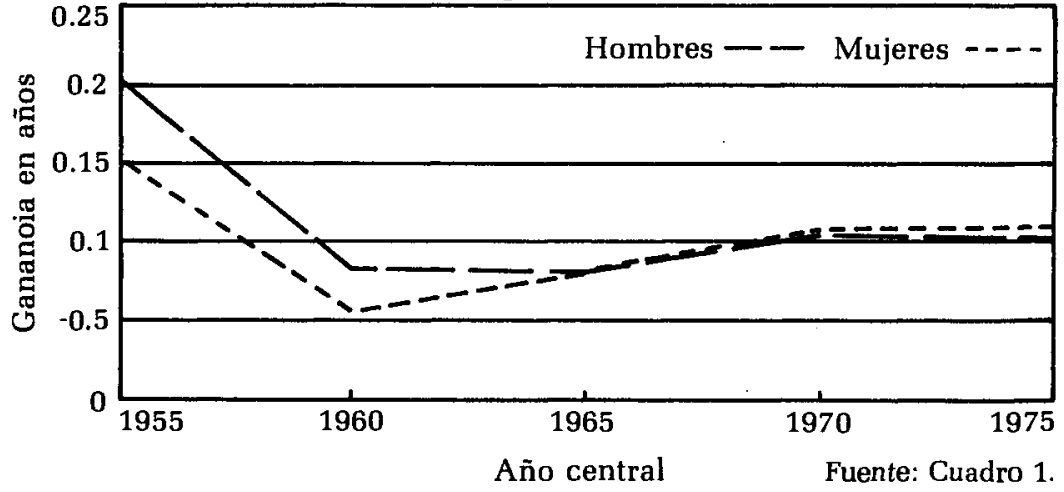


GRÁFICA 13

México: Ganancias en e(55), por sexo, 1950 a 1980

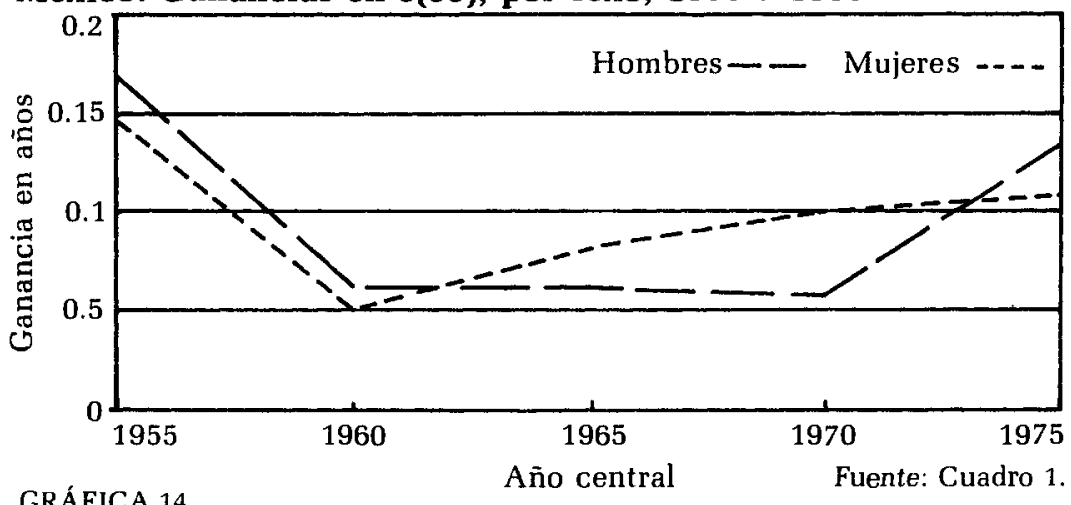

GRÁFICA 14

México: Ganancias en e(60), por sexo, 1950 a 1980

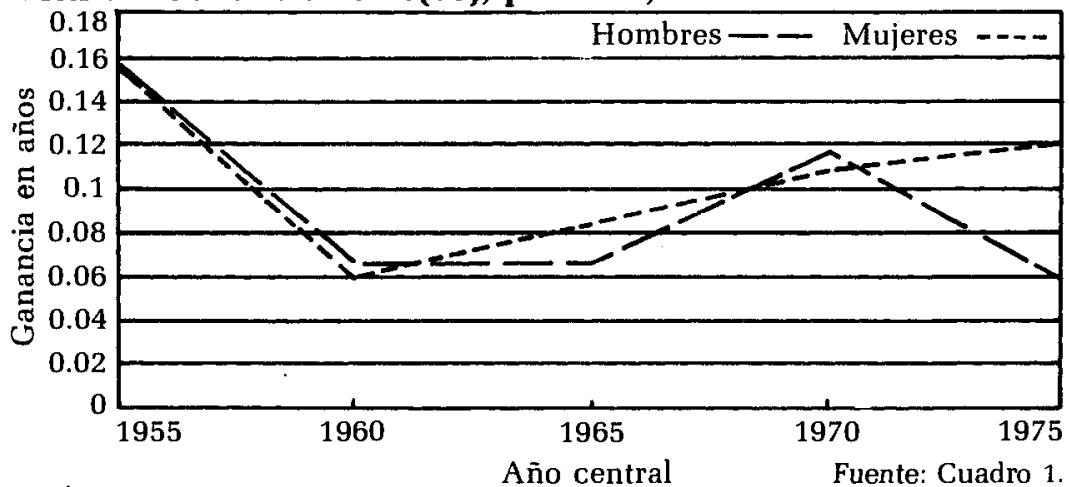

GRÁFICA 15

México: Ganancias en e(65), por sexo, 1950 a 1980

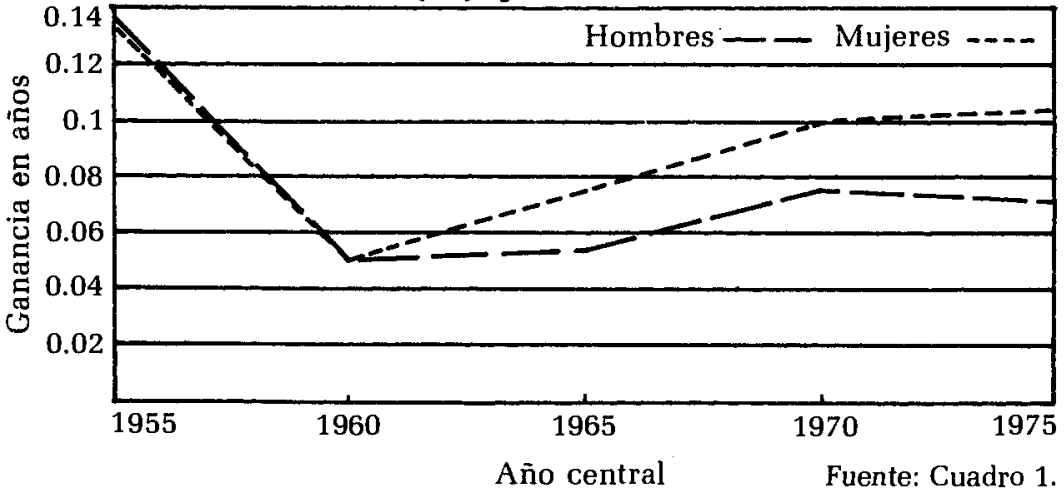


GRÁFICA 16

México: Ganancias en e(70), por sexo, 1950 a 1980

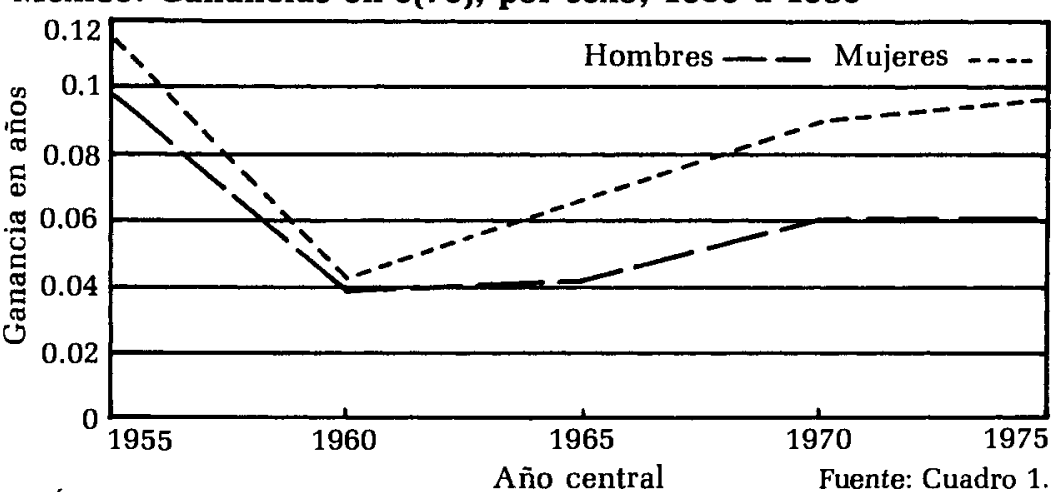

GRÁFICA 17

México: Ganancias en e(75), por sexo, 1950 a 1980

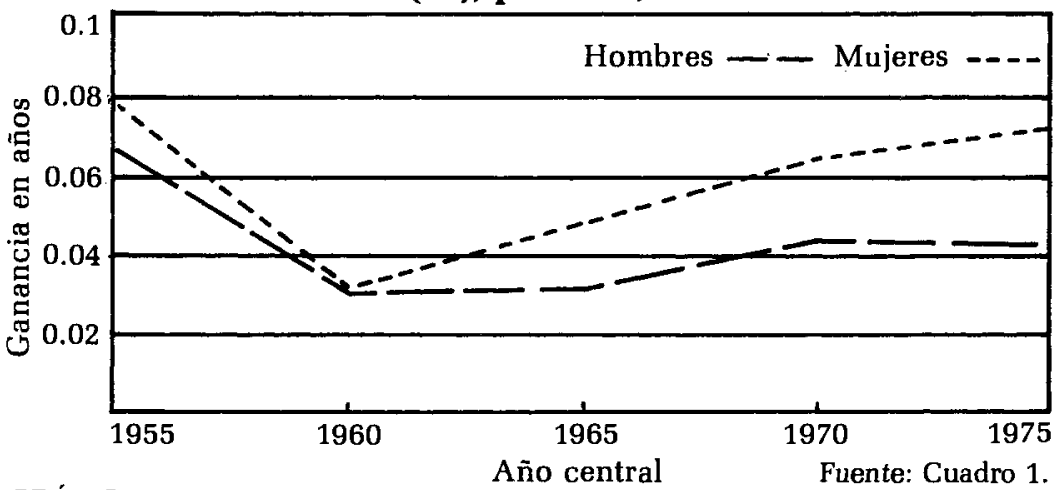

GRÁFICA 18

Año central

México: Ganancias en e(X), por sexo, 1950 a 1980

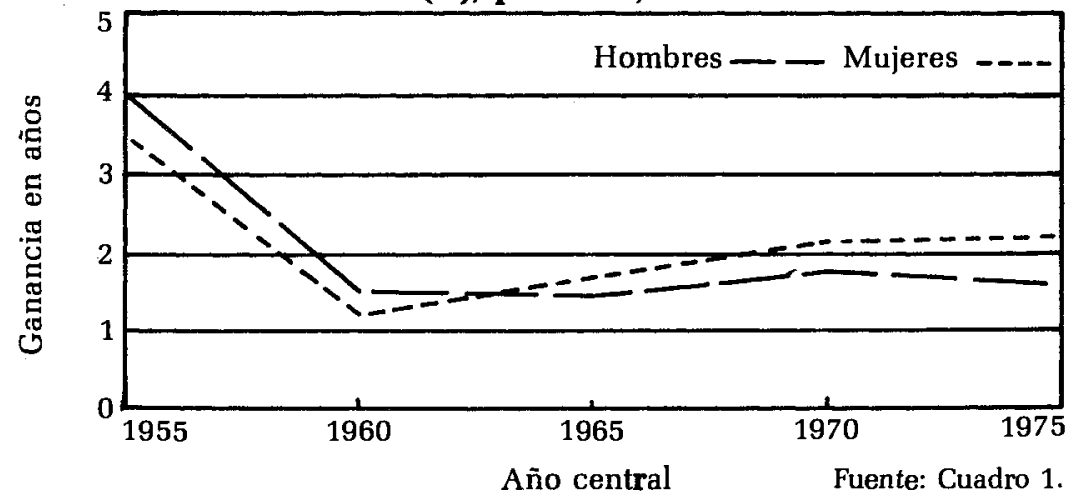

